\title{
The Effect of Gradual Increase in Contextual Interference on Acquisition, Retention and Transfer of Volleyball Skills
}

\author{
Fatemeh Pasand (Corresponding author) \\ Department of physical Education, Shiraz University, Iran \\ E-mail: pasand@shirazu.ac.ir \\ Heydar Fooladiyanzadeh \\ Department of physical Education, Shiraz University, Iran \\ E-mail: hidar.f68@gmail.com \\ Gholamhossien Nazemzadegan \\ Department of physical Education, Shiraz University, Iran \\ E-mail: ghnazem@yahoo.com
}

Received: 18-02-2016

doi:10.7575/aiac.ijkss.v.4n.2p.72
Accepted: 21-04-2016

Published: 30-04-2016

URL: http://dx.doi.org/10.7575/aiac.ijkss.v.4n.2.p.72

\begin{abstract}
Background: A general viewpoint on contextual interference shows that a blocked practice schedule facilitates the acquisition of a skill while a random practice is more useful in the retention and transfer of that skill. Objective: The aim of this study was to investigate the effect of gradual increase in contextual interference upon acquisition, retention and transfer of volleyball skills. Methods: For this purpose, 45 participants were randomly selected from male students at Shiraz University-Iran. After pre-test, the participants were equally distributed in three experimental groups: blocked (low CI), random (high CI) and percentile gradual increase. After nine training sessions and recording the scores, the students were tested for acquisition, retention and transfer. $p \leq 0.05$ was considered as significance level in all the tests. Results: There was significant difference between the groups in acquisition sessions in favor of the blocked group. Retention and transfer test results also showed a significant difference between the groups in favor of random training and gradual increase groups compared to blocked training group .However no significant difference was observed between random with gradual increase training groups. Conclusion: According to the findings of this research, it can be concluded that random and gradual increase in contextual interference training methods may increase the performance of subjects in terms of volleyball skills in retention and transfer tests.
\end{abstract}

Keywords: Gradual Increase of Contextual Interference, Blocked, Random, Volleyball, Acquisition, Retention, Transfer

\section{Introduction}

Education and training conditions are among the most important topics in learning the motor skills. When planning to teach a number of skills in an education and training session, trainers are faced with the question of succession in training students to develop more effective learning conditions (Schmidt \& Lee, 1988). It is very important to organize the trainings to increase their effectiveness. It seems that the exercise of skill is always dependent upon learning; therefore, appropriate training based on principles of motor learning can lead to the storage and retrieval of information in memory, resulting in generation of motor skill according to previously learnt activities (Gallahue \& Ozmun, 2002). Multi-skill training in a training session on the one hand, provides a more attractive environment for the learner and saves time on the other hand. It seems that the use of contextual interference is an approach that satisfies these ends (Porter \& Saemi, 2010). Highlighting the appropriate training program for the learners is a main research objective in learning the motor skills (Schmidt \& lee, 1988). Therefore, one of the primary duties of trainers is to organize the training environment in such a way to improve athletic performance. In this case, two types of training programs in the form of block and random have been proposed. Blocked training is a training stereotype in which the skills are repeated without interference of other activities and all the training efforts of a skill are completed before starting the training of the next skill. In contrast, there is no predefined order of repetitions in random training. Variable training program has been a method for more effective learning of motor skills in recent decades. In planning the variable training, while performing a task, one or several other tasks are also exercised. A method for planning the variable training is taking advantage of a phenomenon known as contextual interference $(\mathrm{CI})$. Contextual interference is a phenomenon whereby interference during learning the skill results in optimal exercise of the skill, which facilitates learning. Blocked and random training methods can bring about different interference levels. Obviously, a combination of blocked and random trainings can be considered as an array of training known as gradual increase. When you are trained in a skill by 
blocked training method, contextual interference is minimal but there is a high level of contextual interference when several different (but interrelated) skills are trained in a randomized training session (Schmidt \& Lee, 1988).

Many studies have indicated that changing random training conditions may lead to poor performance and strong retention and in the same time result in good performance and poor retention in blocked training conditions. It should be noted that in the majority of studies on contextual interference, blocked vs random training has been investigated while there have been few studies on training method of gradual increase in contextual interference and only a few motor skills have been evaluated. In this approach, the training progresses from blocked to random over time with increased comprehensiveness, indicating increased contextual interference. Porter (2008) showed that repeated efforts in the early stages of learning by the learners allows for searching efficient problem-solving strategies, correction of motor errors and development of a basic motor pattern to successfully achieve the planned action. Gradual increase is a new way to provide contextual interference during training sessions in which the training, initially begun with blocked efforts (low contextual interference), is followed by random training (high contextual interference) (Porter, 2008). However, the results of field research in this context are contradictory, so that no effect of contextual interference has been observed in the previous studies in cluding of the study of Meira \& Tani (2001) in assessment of dart-throwing skill, Shewokis (2003) in computer games, Jones and French (2007) in volleyball skills as well as Vera, and Varez \& Medina (2008) in soccer skills.

The majority of studies on contextual interference have dealt with blocked vs random training while few studies have been conducted on training method of gradual increase in contextual interference, which have evaluated only a few motor skills. In this method, the training progresses from blocked to random over time with increased comprehensiveness, which indicates increased contextual interference. On the other hand, there are a few vague evidences concerning the effect of contextual interference in conditions out of the laboratory. Service, set and forearm pass are important skills in volleyball, which have a decisive impact on the outcome of volleyball tournaments. Because of such importance, a large proportion of training sessions are dedicated to these skills by trainers. For a good service, in addition to good physics and expertise, the athlete must have a good understanding of skills. This understanding can include exact knowledge of exercising the skill, indications of exercise and perhaps more importantly, identifying the skills that require further concentration and attention. In this regard, this study aims to evaluate the effect of gradual increase in contextual interference on acquisition, retention and transfer of volleyball skills in three levels of blocked, random and gradual increase.

\section{Methods}

\subsection{Participants}

In this study, 45 male students with mean age of (22.50土1.7) years from Shiraz University in Iran were recruited. They volunteered to participate in the study as well as they had no history of sports activities in volleyball and only received training related to volleyball skills. After pretest the participants were randomly assigned to three groups of 15 subjects, including blocked, random and gradual increase.

\subsection{Task}

Volleyball skills: The volleyball skills in this study included forearm pass, set and service, each performed with a specific guideline (AAHPERD-2 Test) (Antonius and Travlos, 2010).

Simple service: A simple service in which the player hits under the ball towards specified areas with one hand standing behind the line of 9 meters. Set: The subject knuckles to the six areas in the field with a pass coming from a fellow player. Forearm pass: The subject bumps to the six areas in the field with a pass coming from a fellow player.
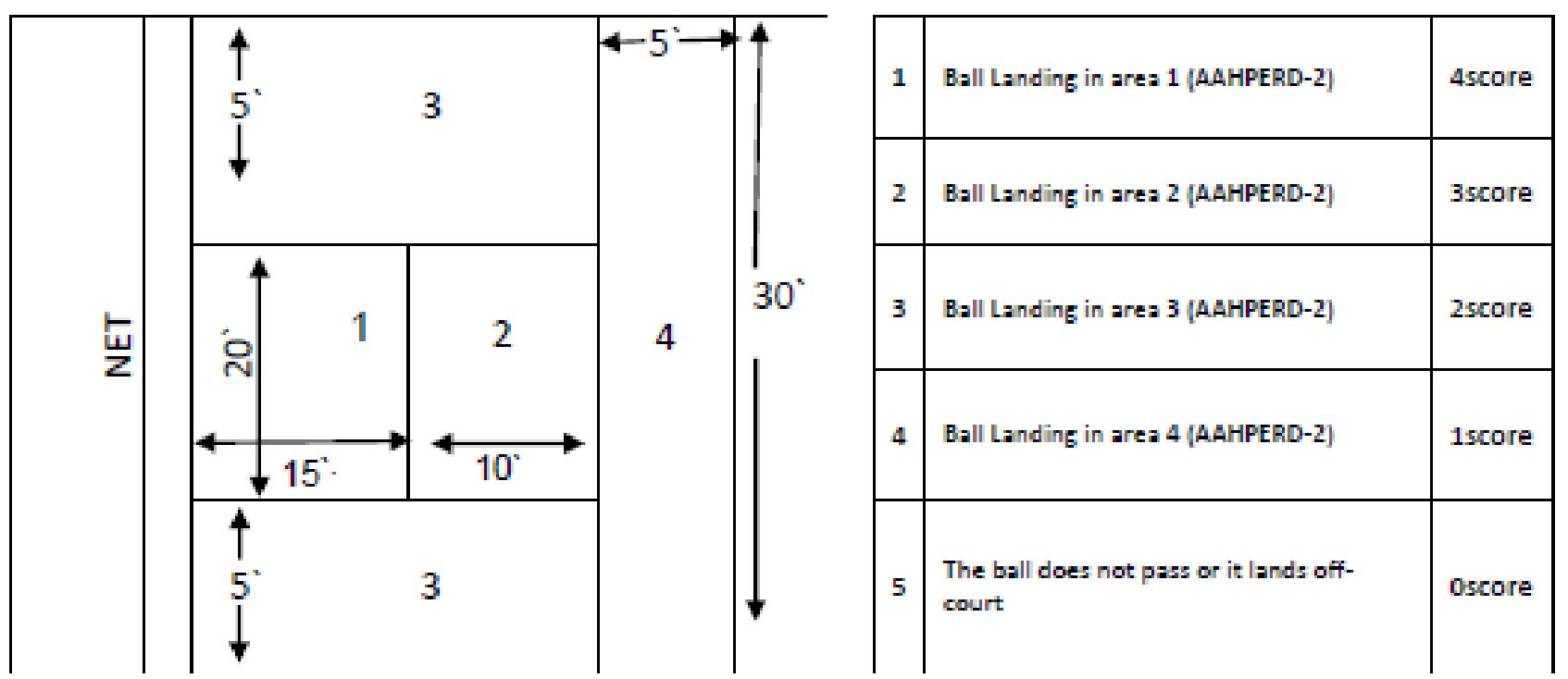

Figure 1. Scores of participants' performance and score ranks in volleyball court (AAHERD-2 test) 
After randomly selecting 45 students as sample from the population, the study steps were first explained for the subjects. After pre-test, they were randomly assigned to three groups of 15 members to match them based on pre-test scores. The groups were trained for skills during three weeks in three sessions per week (nine sessions total).

The first group performed the skills through blocked method. This group was trained for skills in each session using the blocked method hitting 45 trials in each session (15 sets, 15 forearm passes and 15 services).

The second group performed the skills by random method. This group was randomly trained in each session ( 45 hits per session). In this training method, the participants were trained for set, forearm pass and service skills so that none of skills was performed in duplicate (e.g. set, forearm pass, service/forearm pass, set, service/set, service, forearm pass).

The third group performed the skills by gradual increase method. This group was trained for ball throwing skill in each session through gradual increase in contextual interference method. This means that they performed 40 out of 45 training trials by blocked method (14 services, 13 sets, 13 forearm passes) and five trials by random method (set, forearm pass, service, forearm pass, set) in the first session. They performed 35 out of 45 training efforts by blocked method (11 services, 12 sets, 12 forearm passes) and 10 trials by random method (set, forearm pass, service, forearm pass, set, service, forearm pass, set, service, forearm pass) in the second session. Random efforts were increased by $10 \%$ with increasing number of training sessions up to the last session (session 9) until all the skills were randomly performed in the end. The participants were trained for each task during nine sessions (three sessions per week) and three blocks 15 trials for each session (45 trials of all the three skills with a total of 1215 trials).

Acquisition test was performed after the last training session similar to pre-test and training sessions. The scores of skill exercise were recorded after training efforts. Retention test was done 48 hours after the last training session similar to pre-test. Transfer test was done 48 hours after the last training session similar to pre-test with the difference that the transfer test was performed by changing the location of skill exercise. This means that if the participants exercised the skills in the right side of volleyball court during training, they exercised the skills in the left side in the transfer test.

2.4 scoring the performance of participants

By observing the relative threshold of technical matters on the part of subjects, the scoring criterion was recorded for them based on the accuracy of receiving and directing the ball in set and forearm pass as well as landing site of ball in service by the AAHPERD-2 test (Antonius and Travlos, 2010).

\subsection{Data Analyses}

Analysis of variance with repeated measurements as well as ANOVA was used for data analysis. If the difference between groups was significant, post hoc Tukey test was used. All analyzes were performed using SPSS software, version 16 . The significance level was set at 0.05 .

\section{Results}

This experimental study aimed to evaluate the effect of gradual percentile increase in contextual interference on performance and learning of volleyball skills through pre-test, acquisition sessions and post-test, retention and transfer sessions by three training methods (blocked, random and gradual increase). The effect of contextual interference on learning of volleyball skills was designed and developed based on change of skills with different generalized motor program of Magill \& Hall (1990), desired skills according to Bortoli et al (1998) as well as French et al(1990), Jones \& French (2007).

Table 1. Means and standard deviations for the phases and Groups

\begin{tabular}{|c|c|c|c|c|c|c|c|c|}
\hline Phases & \multicolumn{2}{|c|}{ Pre-Test } & \multicolumn{2}{|c|}{ Acquisition } & \multicolumn{2}{|c|}{ Retention } & \multicolumn{2}{|c|}{ Transfer } \\
\hline Groups & M & SD & M & $\mathrm{SD}$ & M & SD & M & SD \\
\hline Blocked & 13 & $2 / 10$ & $33 / 06$ & $1 / 70$ & $26 / 40$ & $2 / 16$ & $22 / 93$ & $2 / 93$ \\
\hline Random & $13 / 42$ & $2 / 24$ & $18 / 35$ & $1 / 33$ & 34 & $1 / 96$ & $35 / 78$ & $2 / 91$ \\
\hline gradual increase & $10 / 40$ & $2 / 92$ & $26 / 40$ & $2 / 16$ & $34 / 46$ & $1 / 95$ & $34 / 66$ & $1 / 98$ \\
\hline
\end{tabular}

With respect to the effect of gradual increase in contextual interference on acquisition, retention and transfer phases of volleyball skills, analysis of variance results with repeated measures and Post-hoc Tukey test showed a significant difference between the study groups $(\mathrm{F}=5.18, \mathrm{p}=0.0001)$. In addition, the Post-hoc Tukey test results showed that the difference was in favor of blocked relative to random $(\mathrm{p}=0 / 015)$. Furthermore, the Tukey test results indicated that gradual increase in contextual interference had no significant effect on acquisition, retention and transfer phases $(\mathrm{p}=0.77, \mathrm{p}=0.069)$.

In the acquisition phase, there was significant difference in $\mathrm{p} \leq 0.05$ confidence level between set, forearms pass and service skills in blocked, random and gradual percentile increase groups $(\mathrm{F}=281.85, \mathrm{p}=0.0001)$. The Post-hoc Tukey test results showed that the difference was in favor of blocked relative to random and gradual increase methods $(p=0 / 0001)$. However, there was no significant difference between random and gradual increase methods $(\mathrm{p}=0.62)$. 
In retention phase of volleyball skills, the difference was in favor of random and gradual increase relative to the blocked training group. A comparison of the results of blocked, random and gradual increase training methods showed a significant difference in confidence level of $\mathrm{p} \leq 0.05$ in retention phase between set, forearm pass and service skills $(\mathrm{F}=73.78, \mathrm{P}=0.0001)$. Moreover, the Post-hoc Tukey test results indicated a significant difference between blocked and gradual increase training methods in retention phase $(\mathrm{p}=0.0001)$.

The results of comparing blocked, random and gradual increase training methods in transfer phase indicated significant difference in confidence level of $\mathrm{p} \leq 0.05$ between set, forearm pass and service skills ( $\mathrm{F}=107.16, \mathrm{P}=0.0001$ ). However, the Post-hoc Tukey test results showed no significant difference between random and gradual increase training methods $(\mathrm{p}=0.496)$.

\section{Discussion}

The aim of this study was to investigate the changes in contextual interference on organization of education and training of volleyball skills through three training methods of blocked, random and gradual increase in order to evaluate and compare learning opportunities of 45 students in three phases of acquisition, retention and transfer. The findings of this study in acquisition phase showed significant differences in confidence level of $\mathrm{p} \leq 0.05$ in favor of blocked relative to random and gradual increase methods in three skills of set, forearm pass and service. In this way, the results were consistent with the results of Smith et al (2003).

Better performance of blocked group relative to random and gradual increase groups is probably due to the absence of interfering tasks as well as exercise of tasks without requirement of new processing in the blocked group. That is because this group prepared a training schedule, which was immediately used in later efforts using short-term memory and caused improved exercise in this phase. However, in the random and gradual increase groups, according to expansion and reconstruction theory of comprehensive action plan, a new task should be designed due to overlap with other tasks. Proponents of expansion theory argue that the development of a training program with high contextual inference (random training) inserts the image of various tasks into the active memory, which in turn results in complete image rich in tasks through the resulting inter- and intra-task processing operations. Reconstruction theory of action plan suggests that in random training program, the action plan used in previous efforts is forgotten as a result of interference with other efforts. The processing activities required for restructuring a forgotten plan of action creates a strong image of skills, which will in turn enhance learning, as a result of which the exercised is abated. In other words, contextual interference causes poor exercise of random and gradual increase methods in the acquisition phase due to implicating the subject in inter- and intra-task processing. Therefore, these perceptual processes and cognitive efforts during acquisition sessions enhance retention and transfer phases. The results of this study are not consistent with the findings of Menayo, Moreno, Sabido (2010), Dias and Mendes (2010), Ollis, Button \& Fairweather (2005), and no effect of contextual interference was observed. These researchers observed no significant difference between the performance of different training groups in various phases of training. Non-compliance of the results of this study can be attributed to difference in field and laboratory tasks as well as differences in the number of training sessions, complexity of the task and expertise level of subjects. On the other hand, Goode \& Magill (1986) stated that one of the reasons for insignificant difference between training groups related to scoring less sensitive in the field tasks rather than laboratory.

Part of the results of this study with respect to observing better effect of blocked training method compared with other training methods in acquisition sessions is consistent with results of Guadagnoli \& Lee (2004), Smith, Gregory \& Davies (2003). In addition, part of these results concerning a significant difference between training groups in retention test was consistent with research conducted by Smith (2002), Saemi, Porter, Ghotbi Varzaneh, Zarghami, \& Shafinia (2012), Porter \& Saemi (2010), Porter (2008), Kalkhoran \& Shariati (2012). Reviewing the literature on contextual interference, Magill \& Hall (1990) reported that when the tasks are exercised under different motor programs, a stronger contextual interference effect is observed relative to when motor programs are the same. This hypothesis of Magill \& Hall on the difference between motor programs and parameter in generation of interference effect has been supported by numerous laboratory studies. While some studies indicate the impact of contextual interference for parameter estimation in retention tests, such impact has not been observed in generalized motor program (GMP) even if the trained tasks are controlled by the same or different parameters (Giuffrida, Shea \& Fairbrother, 2002 and Lai, Shea, Wulf, \& Wright, 2000). Shea \& Morgan (1979) and Shea \& Zimny (1983 and 1988) have suggested that when the training is performed in a random order, there are advantages for learning through interaction between working memory of two or more similar tasks. Increased interference in working memory during training leads to extensive differentiated processing, which eventually facilitates retention. On the other hand, Lee and Magill (1983) and Magill and Hall (1990) argue that interference causes oblivion of action plan in working memory, so these plans are reconstructed in any new effort under random conditions. This reconstruction process causes increased retention and transfer.

While the majority of contextual interference studies have been conducted in laboratory environments, much attention has been recently paid to generalizability of contextual interference results in field environments. The results of field environments are inconsistent and mediocre benefit of high contextual interference has been cited for retention and transfer in laboratory conditions in sports environments because of features such as complexity of skills and expertise level of the learner. In applied research, several skills of various sports activities have been studied and the results are still incompatible with each other. For example, the results of studies with the same tasks controlled by a motor program requiring parameter adjustments are inconsistent. For instance, the results of this study are not consistent with 
Chamberlin (1991) jump shot in basketball as well as Hall and Boyle (1993) on shuffleboard skill, which reported no significant difference between training arrangement methods. The results are also inconsistent with those of Meira \& Tani (2001) in acquisition of dart -throwing skill. On the other hand, the results of this study in acquisition phase were not consistent with studies conducted by Rouhollahi, Rozan \& Mehrotra (2014) and Afsanepurak, Karimiyani, Moradi \& Safaei (2012). Landin and Hebert (1997) reported no significant difference between training methods in acquisition of basketball shooting skills.

However, there are limitations in this study that should be considered in future research. For example, positive results can be obtained by increasing the sample size or increasing the efforts related to trainings.

According to the results of this study, it can be generally concluded that contextual interference can lead to learning of motor skills in blocked, random and gradual percentile increase in contextual interference methods. Overall, skill training methods should be designed considering several factors such as age, expertise and experience level of subjects, motor and cognitive abilities, processing capacity of environmental information and several other factors that can affect motor learning, and there should always be an appropriate optimal structure between pervasive expertise level and difficulty of training skills.

\section{Conclusion}

The results support the claim that training with contextual interference in both aspects of memory power in connection with expansion, differentiation and reconstruction hypothesis of action plan causes progress with respect to forgetting and reconstruction hypothesis. Although it generally seems that random and gradual percentile increase training methods are superior for learning, more research is needed to confirm this matter. Moreover, according to the results obtained with respect to higher impact of gradual increase and random methods relative to blocked method, the application of these training methods is suggested in order to educate motor skills in different age ranges.

\section{Acknowledgments}

We are thankful students of Shiraz University for volunteering their time to participate.

\section{References}

Afsanepurak, S. A., Karimiyani, N., Moradi, J., \& Safaei, M. (2012). The Effect of Blocked, Random, and Systematically Increasing Practice on learning of Different Types of Basketball Passes. European Journal of Experimental Biology, 2(6), 2397-2402 .

Antonius, K., Travlos. (2010) (1). "Specificity and variability of practice, and contextual interference in acquisition and transfer of an underhand volleyball serve”. Perceptual and Motor Skills. Vol. 110, Issue, pp: 298-312.

Bortoli, L., Robazza, C., Durigon, V., \& Carra, C. (1992). Effects of contextual interference on learning technical sports skills. Perceptual and motor skills, 75(2), 555-562.

Chamberlin, C., Rimer, T., \& Skaggs, D. (1990). The ecological validity of the contextual interference effect: A practical application to learning the jump shot in basketball. Paper presented at the annual meeting of the North American Society for the Psychology of Sport and Physical Activity. Houston, TX, May.

Dias, G., \& Mendes, R. (2010). Effects of a contextual interference continuum on golf putting task. Revista Brasileira de Educação Física e Esporte, 24(4), 545-553.

Feghhi, I., Valizadeh, R., Rahimpour, M., Tehrani, M. A., \& Karampour, S. (2015). Contextual Interference in Learning Three Table Tennis Services. Procedia-Social and Behavioral Sciences, 191, 546-549.

French, K. E., Rink, J. E., \& Werner, P. H. (1990). Effects of contextual interference on retention of three volleyball skills. Perceptual and motor skills, 71(1), 179-186.

Gallahue, D., \& Ozmun, J. (2002). Understanding motor development: Infants, children, adolescents, adults with PowerWeb .

Giuffrida, C. G., Shea, J. B., \& Fairbrother, J. T. (2002). Differential transfer benefits of increased practice for constant, blocked, and serial practice schedules. Journal of motor behavior, 34(4), 353-365 .

Goode, S., \& Magill, R. A. (1986). Contextual interference effects in learning three badminton serves. Research quarterly for exercise and sport, 57(4), 308-314 .

Guadagnoli, M. A \& ,.Lee, T. D. (2004). Challenge point: a framework for conceptualizing the effects of various practice conditions in motor learning. Journal of motor behavior, 36(2), 212-224 .

Hall, K., \& Boyle, M. (1993). The effects of contextual interference on shuffleboared skill in children. Research quarterly for exercise and sport, 67(1), 52-58 .

Jones, L. L., \& French, K. E. (2007). Effects of contextual interference on acquisition and retention of three volleyballskills 1. Perceptual and motor skills, 105(3), 883-890. 
Kalkhoran, A. F., \& Shariati, A. (2012). The Effects of Contextual Interference on Learning Volleyball Motor Skills. Journal of Physical Education and Sport, 12(4), 550 .

Lai, Q., Shea, C. H., Wulf, G., \& Wright, D. L. (2000). Optimizing generalized motor program and parameter learning. Research quarterly for exercise and sport, 71(1), 10-24 .

Landin, D., \& Hebert, E. P. (1997). A comparison of three practice schedules along the contextual interference continuum. Research quarterly for exercise and sport, 68(4), 357-361 .

Lee, T. D., \& Magill, R. A. (1983). The locus of contextual interference in motor-skill acquisition. Journal of Experimental Psychology: Learning, Memory, and Cognition, 9(4), 730 .

Magill, R. A., \& Hall, K. G. (1990). A review of the contextual interference effect in motor skill acquisition. Human movement science, 9(3), 241-289.

Meira Jr, C. M., \& Tani, G. (2001). The contextual interference effect in acquisition of dart-throwing skill tested on a transfer test with extended trials. Perceptual and motor skills, 92(3), 910-918.

Menayo, R., Moreno, F., Sabido, R., Fuentes, J., \& Garcia, J. (2010). Simultaneous treatment effects in learning four tennis shots in contextual interferenceconditions1, 2. Perceptual and motor skills, 110(2), 661-673.

Ollis, S., Button, C., \& Fairweather, M. (2005). The influence of professional expertise and task complexity upon the potency of the contextual interference effect. Acta Psychologica, 118(3), 229-244 .

Porter, J. M. (2008). Systematically increasing contextual interference is beneficial for learning novel motor skills .

Porter, J. M., \& Saemi, E. (2010). Moderately skilled learners benefit by practicing with systematic increases in contextual interference. International Journal of Coaching Science, 4(2), 61-71 .

Rouhollahi,V. Rozan,M. Mehrotra,A.(2014). Effect of Different Practice Schedules on Learning and Performance in Handball Task, American Journal of Sports Science. Vol. 2, No. 4, 2014, pp. 71-76.

Saemi, E., Porter, J. M., Ghotbi Varzaneh, A., Zarghami, M., \& Shafinia, P. (2012). Practicing along the contextual interference continuum: A comparison of three practice schedules in an elementary physical education setting . Kineziologija, 44(2), 191-198 .

Schmidt, R. A., \& Lee, T. (1988). Motor control and learning .

Shea, J. B., \& Morgan, R. L. (1979). Contextual interference effects on the acquisition, retention, and transfer of a motor skill. Journal of Experimental Psychology: Human Learning and Memory, 5(2), 179 .

Shea, J. B., \& Zimny, S. T. (1983). Context effects in memory and learning movement information. Advances in Psychology, 12, 345-366.

Shea, J. B., \& Zimny, S. T. (1988). Knowledge incorporation in motor representation. Advances in Psychology, 50, 289314 .

Shewokis, P. A. (2003). Memory consolidation and contextual interference effects with computer games. Perceptual and motor skills, 97(2), 581-589.

Smith, P. J. (2002). Applying contextual interference to snowboarding skills. Perceptual and motor skills, 95(3), 9991005 .

Smith, P. J., Gregory, S. K., \& Davies, M. (2003). Alternating versus blocked practice in learning a cartwheel. Perceptual and motor skills, 96(3c), 1255-1264. 